\title{
Calidad de vida y felicidad percibida en estudiantes mujeres de Lima Centro
}

\author{
Quality of life and happiness perceived in female students of Lima Center \\ Daniela Barraza-Mendoza ${ }^{1}$ \\ María Flores-Aime ${ }^{2}$
}

\begin{abstract}
Resumen
Objetivo: determinar la relación entre la calidad de vida y la felicidad percibida en estudiantes mujeres de Lima Centro. Materiales y métodos: el estudio corresponde al tipo correlacional de corte transversal. La muestra estuvo conformada por 245 estudiantes de sexo femenino, de tercero a quinto grado de secundaria. Los instrumentos que se utilizaron fueron la Escala de Calidad de vida de Olson \& Barnes, la Escala de Felicidad de Lima y una ficha de datos sociodemográficos. Para el análisis estadístico se utilizó el programa Stata 15. En cuanto al análisis descriptivo, se calcularon frecuencias, porcentajes, promedio y desviación estándar. Por otro lado, para el análisis bivariado se utilizaron las pruebas de ShapiroWilk, Chi-Cuadrado y ANOVA. Resultados: se evidenció una relación significativa entre la felicidad y la calidad de vida. Asimismo, se encontró relación entre la felicidad con las dimensiones de la calidad de vida: hogar y bienestar económico ( $\mathrm{p}=0.000)$, amigos, vecindario y comunidad $(\mathrm{p}=0.000)$, vida familiar y familia extensa $(\mathrm{p}=0.000)$, educación y ocio $(\mathrm{p}=0.000)$, medios de comunicación $(\mathrm{p}=0.017)$, religión $(\mathrm{p}=0.001)$ y salud $(\mathrm{p}=0.000)$. Conclusión: la calidad de vida se relaciona con la felicidad. Esto indica que en determinados contextos las adolescentes perciben que su deficiente calidad de vida interfiere con su estado de felicidad. Sin embargo, debido a la etapa en la que se encuentran los cambios biológicos, psicológicos y culturales pueden variar notablemente su percepción.
\end{abstract}

Palabras clave: Calidad de vida; Felicidad; Mujeres; Adolescente; Religión (Fuente: DeCS).

\begin{abstract}
Objective: to determine the relationship between the quality of life and the happiness perceived in female students of Lima Center. Materials and methods: the study corresponds to the cross-sectional correlational type. The sample consisted of 245 female students, from third to fifth grade of secondary school. The instruments that were used were the Olson \& Barnes Quality of Life Scale, the Lima Happiness Scale and a sociodemographic data sheet. For the statistical analysis, the Stata 15 program was used. As for the descriptive analysis, frequencies, percentages, average and standard deviation were calculated. On the other hand, the Shapiro-Wilk, Chi-Square and ANOVA tests were used for the bivariate analysis. Results: a significant relationship between happiness and quality of life was evident. Likewise, a relationship was found between happiness and the dimensions of quality of life: home and economic well-being $(\mathrm{p}=0.000)$, friends, neighborhood and community $(\mathrm{p}=0.000)$, family life and extended family $(\mathrm{p}=0.000)$, education and leisure $(\mathrm{p}=0.000)$, media $(\mathrm{p}=0.017)$, religion $(\mathrm{p}=0.001)$ and health $(\mathrm{p}=0.000)$. Conclusion: the quality of life is related to happiness. This indicates that in certain contexts adolescents perceive that their poor quality of life interferes with their state of happiness. However, due to the stage in which biological, psychological and cultural changes are found, their perception can vary significantly.
\end{abstract}

Keys words: Quality of life; Happiness; Women; Adolescent; Religion (Source: DeCS).

Para citar:

Barraza D, Flores M. Calidad de vida y felicidad percibida en estudiantes mujeres de Lima Centro. CASUS. 2019;4(3):153-161.

DOI: $10.35626 /$ casus.3.2019.216

\footnotetext{
${ }^{1}$ Universidad Católica Sedes Sapientiae.

${ }^{2}$ Puesto Parroquial de Salud Mental San José.

Correo electrónico: daniebarrazam@gmail.com
}

Fecha de recepción: 21-06-19

Fecha de envío a pares: 24-06-19

Fecha de aprobación por pares: 11-11-19

Fecha de aceptación: 16-11-19 


\section{INTRODUCCIÓN}

A nivel mundial aproximadamente 450 millones de personas padecen un trastorno psicopatológico o de la conducta, de los cuales el $20 \%$ de esta población son niños y adolescentes (1). Esta situación genera efectos relacionados al bienestar social, la calidad de vida y al nivel emocional (1). De hecho, estos efectos son la manifestación de estados emocionales negativos que se caracterizan por la insatisfacción con la vida, desánimo, sufrimiento, tristeza, culpa, sentimientos de odio y estrés (2); de manera que limitan la funcionalidad en las actividades de la vida diaria (2). Asimismo, estos factores psicoemocionales que afectan la calidad de vida de la persona, podrían estar relacionados con una disminución de la felicidad que es el estado de satisfacción plena que experimenta subjetivamente el individuo por un bien deseado (3).

Al respecto, dentro del contexto mundial, se ha despertado el interés por conocer los factores involucrados en la felicidad. Precisamente, en este año se han identificado 13773 publicaciones sobre el tema (4). Por ejemplo, The World Happiness Report (WHR) clasificó a 156 países según sus niveles de felicidad y el Perú ocupa el puesto 65. Mientras que, a nivel de Sudamérica se encuentra al final de esta lista solo encima de Venezuela que está en el puesto 108 (5). Por su parte, una investigación realizada en todos los departamentos del Perú reportó que la ciudad más feliz era Huancayo, identificando que los individuos de esta región se percibían más felices debido a tres factores referentes a la calidad de vida: la familia, un buen lugar para vivir y la aceptación de sí mismo (6). Es decir, que la felicidad estaría relacionada a la calidad de vida.

La calidad de vida, se considera como un conjunto de dominios objetivos y subjetivos que incluyen el hogar, la salud, el ingreso económico, la vida social y la salud mental (7). De esta manera, se asocia con la felicidad, ya que el sujeto percibirá su estado actual de manera positiva o negativa en función de las vivencias y los recursos que posee. Por ende, quienes presentan una estabilidad en aspectos como la salud, la educación, las relaciones interpersonales, la realización de actividades religiosas y la seguridad que proporciona la familia, podrían indicar experiencias de felicidad en sus vidas. Por el contrario, la insatisfacción en estos factores que involucra la calidad de vida puede generar una felicidad no alcanzada (8).

Por lo tanto, se puede considerar que la calidad de vida y la felicidad son aspectos fundamentales que promueven el desarrollo integral y la productividad en las diferentes áreas de vida del adolescente. De igual manera, las diferentes actividades que le hacía feliz cuando era niño van cambiando conforme el adolescente crece y se evidencia en los aspectos de la calidad de vida como el apoyo familiar, la competencia social y la autorregulación que son considerados predictores de la felicidad $(9,10)$.

En este sentido, existen investigaciones a nivel nacional que indagan sobre la relación entre la felicidad y la calidad de vida. Sin embargo, la mayoría de estas se han orientado en muestras mixtas, sin tomar en cuenta específicamente una población escolar femenina. Por otra parte, profundizar en esta relación resultaría relevante ya que los resultados podrían contribuir a identificar qué aspectos de la calidad de vida favorecen un estado de felicidad en estas adolescentes que están próximas a formar parte de la ciudadanía y por ende, la contribución que aporten a la sociedad dependerá en la medida de cómo ellas se sientan y se perciban en su medio social. Por tal motivo, el objetivo principal de la presente investigación fue determinar la relación entre la calidad de vida y la felicidad percibida en estudiantes mujeres de Lima Centro.

\section{MATERIALES Y MÉTODOS}

El tipo de estudio fue de alcance correlacional y el diseño de corte transversal. La muestra estuvo conformada por 245 estudiantes de sexo femenino matriculadas del 3ro al 5to grado del nivel secundario de una Institución Educativa de Breña, 
seleccionadas a través del muestreo probabilístico bietápico estratificado.

Las variables principales fueron: calidad de vida y felicidad. La calidad de vida, se define como un juicio subjetivo del grado en que se ha alcanzado la felicidad, la satisfacción, o como un sentimiento de bienestar personal (11). Es una variable cualitativa politómica ordinal, y fue medida a través de la Escala de calidad de vida de Olson y Barnes, se categorizó (de manera general) en: calidad de vida óptima (86 a más), tendencia a calidad de vida buena (54 a 85), tendencia a baja calidad de vida (16 a 53) y mala calidad de vida (menos de 16) (12). Asimismo, las dimensiones para medir la calidad de vida son: hogar $\mathrm{y}$ bienestar económico, amigos, vecindario y comunidad, vida familiar y familia extensa, educación y ocio, medios de comunicación, religión, salud (8).

Por otro lado, la felicidad se define como un proceso de satisfacción que un individuo percibe y experimenta de manera subjetiva por un bien deseado (13). La felicidad es una variable cualitativa politómica ordinal, fue medida con la Escala de Felicidad de Lima y se categoriza en cinco niveles: muy baja felicidad (27 a 87), baja felicidad (88 a 95), felicidad media (96 a 110), alta felicidad (111 a 118) y muy alta felicidad (119 a 135). Asimismo, las dimensiones de la variable felicidad son: sentido positivo de la vida, satisfacción con la vida, realización personal y alegría de vivir (13).

Respecto a las variables sociodemográficas se utilizó una ficha sociodemográfica en la cual se consideraron: edad, variable cuantitativa con una escala de razón; grado escolar, variable cualitativa politómica ordinal (tercero, cuarto y quinto); ocupación, dicotómica nominal (estudiante, trabaja); tipo de vivienda, dicotómica nominal (propia, alquilada); con quienes vive, politómica nominal (solo padre, solo madre, ambos padres, solo familiares, padres y familiares, solo).
Para el análisis de datos se utilizó el paquete estadístico Stata versión 15. A nivel descriptivo, las variables cualitativas fueron medidas a través de frecuencias y porcentajes. En el caso de la variable cuantitativa se utilizó una medida de tendencia central (promedio) y de dispersión (desviación estándar). Respecto al análisis bivariado se utilizó la prueba de normalidad de Shapiro Wilk y para hallar la relación entre las variables se usó Chi-cuadrado y ANOVA considerando niveles de significancia estadística con un valor de 0.05 .

\section{RESULTADOS}

De las 245 estudiantes de secundaria, la edad promedio fue de 15.4 años, el $37.96 \%$ fue de tercero de secundaria, el $97.55 \%$ no trabajaba al momento de la encuesta, el $60.82 \%$ reportó vivir en una casa propia y el $33.88 \%$ manifestó tener una familia con ambos padres. Asimismo, el $36.73 \%$ presentó tendencia a baja calidad de vida. Por otro lado, el $31.02 \%$ reportó muy baja felicidad. El resto de resultados se puede visualizar en la tabla 1.

Tabla 1. Descripción de las variables en las estudiantes de secundaria

\begin{tabular}{lcc}
\hline Variables & n & \% \\
\hline Edad $(\mathrm{m} \pm$ DS) & 15.4 & 1.127 \\
Grado & & \\
$\quad$ Tercero de secundaria & 93 & 37.96 \\
Cuarto de secundaria & 75 & 30.61 \\
$\quad$ Quinto de secundaria & 77 & 31.43 \\
Ocupación & & \\
$\quad$ Estudiante & 239 & 97.55 \\
$\quad$ Trabaja & 6 & 2.45 \\
Tipo de vivienda & & \\
Propia & 149 & 60.82 \\
Alquilada & 96 & 39.18 \\
Con quienes vive & & \\
$\quad$ Solo padre & 5 & 2.04 \\
$\quad$ Solo madre & 68 & 27.76 \\
Ambos padres & 83 & 33.88 \\
$\quad$ Solo familiares & 33 & 13.47 \\
Padres y familiares & 54 & 22.04 \\
$\quad$ Solo & 2 & 0.82 \\
Calidad de vida & & \\
Calidad de vida óptima & 29 & 11.84 \\
Tendencia a calidad de vida buena & 56 & 22.86 \\
Tendencia a baja calidad de vida & 90 & 36.73 \\
Mala calidad de vida & 70 & 28.57 \\
\hline
\end{tabular}


En la tabla 2, se aprecia una relación significativa entre la felicidad y la calidad de vida $(\mathrm{p}=0.000)$; es decir el $60 \%$ de las estudiantes que reportó mala calidad de vida presentó muy baja felicidad. Asimismo, se encontró relación entre la felicidad con las dimensiones de la calidad de vida: hogar y bienestar económico $(\mathrm{p}=0.000)$, amigos, vecindario y comunidad $(\mathrm{p}=0.000)$, vida familiar $\mathrm{y}$ familia extensa $(\mathrm{p}=0.000)$, educación y ocio $(\mathrm{p}=0.000)$, medios de comunicación $(\mathrm{p}=0.017)$, religión $(\mathrm{p}=0.001)$ y salud $(\mathrm{p}=0.000)$. Las demás variables no mostraron significancia por el cual no fueron incluidas en la tabla.

Tabla 1. continuación

\begin{tabular}{|c|c|c|}
\hline Variables & $\mathbf{n}$ & $\%$ \\
\hline \multicolumn{3}{|l|}{$\begin{array}{l}\text { Hogar y bienestar } \\
\text { económico }\end{array}$} \\
\hline Calidad de vida óptima & 128 & 52.24 \\
\hline $\begin{array}{l}\text { Tendencia a calidad de } \\
\text { vida buena }\end{array}$ & 60 & 24.49 \\
\hline $\begin{array}{l}\text { Tendencia a baja calidad } \\
\text { de vida }\end{array}$ & 52 & 21.22 \\
\hline $\begin{array}{l}\text { Mala calidad de vida } \\
\text { Amigos, vecindario y } \\
\text { comunidad }\end{array}$ & 5 & 2.04 \\
\hline Calidad de vida óptima & 18 & 7.35 \\
\hline $\begin{array}{l}\text { Tendencia a calidad de } \\
\text { vida buena }\end{array}$ & 58 & 23.67 \\
\hline $\begin{array}{l}\text { Tendencia a baja calidad } \\
\text { de vida }\end{array}$ & 105 & 42.86 \\
\hline Mala calidad de vida & 64 & 26.12 \\
\hline $\begin{array}{l}\text { Vida familiar y familia } \\
\text { extensa }\end{array}$ & & \\
\hline Calidad de vida óptima & 169 & 68.98 \\
\hline $\begin{array}{l}\text { Tendencia a calidad de } \\
\text { vida buena }\end{array}$ & 27 & 11.02 \\
\hline $\begin{array}{l}\text { Tendencia a baja calidad } \\
\text { de vida }\end{array}$ & 37 & 15.1 \\
\hline Mala calidad de vida & 12 & 4.90 \\
\hline Educación y ocio & & \\
\hline Calidad de vida óptima & 29 & 11.84 \\
\hline $\begin{array}{l}\text { Tendencia a calidad de } \\
\text { vida buena }\end{array}$ & 100 & 40.82 \\
\hline $\begin{array}{l}\text { Tendencia a baja calidad } \\
\text { de vida }\end{array}$ & 69 & 28.16 \\
\hline Mala calidad de vida & 47 & 19.18 \\
\hline Medios de comunicación & & \\
\hline Calidad de vida óptima & 119 & 48.57 \\
\hline $\begin{array}{l}\text { Tendencia a calidad de } \\
\text { vida buena }\end{array}$ & 67 & 27.35 \\
\hline $\begin{array}{l}\text { Tendencia a baja calidad } \\
\text { de vida }\end{array}$ & 47 & 19.18 \\
\hline Mala calidad de vida & 12 & 4.90 \\
\hline
\end{tabular}

Tabla 1. continuación

\begin{tabular}{|c|c|c|}
\hline \multirow{2}{*}{\multicolumn{3}{|c|}{$\begin{array}{l}\text { Variables } \\
\text { Religión }\end{array}$}} \\
\hline & & \\
\hline Calidad de vida óptima & 38 & 15.51 \\
\hline $\begin{array}{l}\text { Tendencia a calidad de } \\
\text { vida buena }\end{array}$ & 49 & 20.00 \\
\hline $\begin{array}{l}\text { Tendencia a baja calidad } \\
\text { de vida }\end{array}$ & 95 & 38.78 \\
\hline Mala calidad de vida & 63 & 25.71 \\
\hline Salud & & \\
\hline Calidad de vida óptima & 49 & 20.00 \\
\hline $\begin{array}{l}\text { Tendencia a calidad de } \\
\text { vida buena }\end{array}$ & 86 & 35.10 \\
\hline $\begin{array}{l}\text { Tendencia a baja calidad } \\
\text { de vida }\end{array}$ & 82 & 33.47 \\
\hline $\begin{array}{l}\text { Mala calidad de vida } \\
\text { Felicidad }\end{array}$ & 28 & 11.43 \\
\hline Muy baja felicidad & 76 & 31.02 \\
\hline Baja felicidad & 28 & 11.43 \\
\hline Felicidad media & 68 & 27.76 \\
\hline Alta felicidad & 33 & 13.47 \\
\hline Muy alta felicidad & 40 & 16.33 \\
\hline
\end{tabular}

Por otro lado, se evidenció relación significativa entre las personas con quienes viven en el hogar y la felicidad $(\mathrm{p}=0.017)$, donde el $36.76 \%$ de las alumnas que reportó vivir solo con la madre presentó niveles de felicidad media. Ver tabla 3.

\section{DISCUSIÓN}

Los resultados mostraron que existe relación significativa entre la calidad de vida y la felicidad. Además, se evidenció relación entre las dimensiones de calidad de vida con los niveles de felicidad. De igual forma, se halló relación entre la felicidad y con quienes viven las estudiantes.

Las adolescentes que reportaron una mala calidad de vida presentaron un nivel muy bajo de felicidad. Otras investigaciones corroboran este resultado $(14,15,16,17)$. Una posible explicación de los resultados es que las estudiantes que presentaron mala calidad de vida poseen una percepción negativa sobre su situación actual (8). Asociado a una insatisfacción en las diferentes experiencias relacionadas a los dominios que involucra la calidad de vida tales como el hogar, el ingreso económico, la salud, la vida social, la vida familiar. Ya que estos aspectos vistos negativamente pueden conllevar a percibir una felicidad no alcanzada (8). 
Tabla 2. Asociación entre calidad de vida y felicidad

\begin{tabular}{|c|c|c|c|c|c|c|}
\hline & & Felici & & & & \\
\hline & $\begin{array}{c}\text { Muy baja } \\
\text { felicidad } \\
\text { n }(\%)\end{array}$ & $\begin{array}{c}\text { Baja } \\
\text { felicidad } \\
\text { n }(\%)\end{array}$ & $\begin{array}{c}\text { Felicidad } \\
\text { media } \\
\text { n }(\%)\end{array}$ & $\begin{array}{c}\text { Alta } \\
\text { felicidad } \\
\text { n }(\%)\end{array}$ & $\begin{array}{c}\text { Muy alta } \\
\text { felicidad } \\
\text { n (\%) }\end{array}$ & $\mathrm{P}$ \\
\hline Calidad de vida gener & & & & & & 0.000 \\
\hline $\begin{array}{l}\text { Calidad de vida } \\
\text { óptima }\end{array}$ & $0(0.00)$ & $2(6.90)$ & $3(10.34)$ & $5(17.24)$ & $19(65.52)$ & \\
\hline $\begin{array}{l}\text { Tendencia a calidad } \\
\text { de vida buena }\end{array}$ & $7(12.50)$ & $4(7.14)$ & $21(37.50)$ & $14(25.00)$ & $10(17.86)$ & \\
\hline $\begin{array}{l}\text { Tendencia a baja } \\
\text { calidad de vida }\end{array}$ & $27(30.00)$ & $9(10.00)$ & $33(36.67)$ & $14(15.56)$ & $7(7.78)$ & \\
\hline Mala calidad de vida & $42(60.00)$ & $13(18.57)$ & $11(15.71)$ & $0(0.00)$ & $4(5.71)$ & \\
\hline Hogar y bienestar eco & lómico & & & & & 0.000 \\
\hline $\begin{array}{l}\text { Calidad de vida } \\
\text { óptima }\end{array}$ & $20(15.63)$ & $12(9.38)$ & $42(32.81)$ & $23(17.97)$ & $31(24.22)$ & \\
\hline $\begin{array}{l}\text { Tendencia a calidad } \\
\text { de vida buena }\end{array}$ & $26(43.33)$ & $7(11.67)$ & $14(23.33)$ & $8(13.33)$ & $5(8.33)$ & \\
\hline $\begin{array}{l}\text { Tendencia a baja } \\
\text { calidad de vida }\end{array}$ & $27(51.92)$ & $9(17.31)$ & $10(19.23)$ & $2(3.85)$ & 4(7.69) & \\
\hline Mala calidad de vida & $3(60.00)$ & $0(0.00)$ & $2(40.00)$ & $0(0.00)$ & $0(0.00)$ & \\
\hline $\begin{array}{l}\text { Amigos, vecindario y } \\
\text { Calidad de vida }\end{array}$ & omunidad & & & & & 0.000 \\
\hline $\begin{array}{l}\text { óptima } \\
\text { Tendencia a calidad }\end{array}$ & $1(5.56)$ & $1(5.56)$ & $4(22.22)$ & $2(11.11)$ & $10(55.56)$ & \\
\hline $\begin{array}{l}\text { de vida buena } \\
\text { Tendencia a baja }\end{array}$ & $8(13.79)$ & $3(5.17)$ & $21(36.21)$ & $16(27.59)$ & $10(17.24)$ & \\
\hline $\begin{array}{l}\text { 1endencia a baja } \\
\text { calidad de vida }\end{array}$ & $32(30.48)$ & $14(13.33)$ & $35(33.33)$ & $9(8.57)$ & $15(14.29)$ & \\
\hline Mala calidad de vida & $35(54.69)$ & $10(15.63)$ & $8(12.50)$ & $6(9.38)$ & $5(7.81)$ & \\
\hline Vida familiar y famili & extensa & & & & & 0.000 \\
\hline $\begin{array}{l}\text { Calidad de vida } \\
\text { óptima }\end{array}$ & $34(20.12)$ & $18(10.65)$ & $49(28.99)$ & $31(18.34)$ & $37(21.89)$ & \\
\hline $\begin{array}{l}\text { Tendencia a calidad } \\
\text { de vida buena }\end{array}$ & $11(40.74)$ & $6(22.22)$ & $8(29.63)$ & $2(7.41)$ & $0(0.00)$ & \\
\hline $\begin{array}{l}\text { Tendencia a baja } \\
\text { calidad de vida }\end{array}$ & $23(62.16)$ & $2(5.41)$ & $10(27.03)$ & $0(0.00)$ & $2(5.41)$ & \\
\hline Mala calidad de vida & $8(66.67)$ & $2(16.67)$ & $1(8.33)$ & $0(0.00)$ & $1(8.33)$ & \\
\hline
\end{tabular}

En cuanto a las dimensiones de la calidad de vida, se observó en las adolescentes que evidenciaron un nivel óptimo en hogar y bienestar económico presentaron un nivel medio de felicidad. De igual manera, este hallazgo coincide con otros estudios $(14,16)$. Una probable explicación podría relacionarse a la presencia de una buena economía en el hogar, debido a que facilita las comodidades como: los bienes materiales, la vestimenta, la educación, la salud, entre otros (18). Aunque el ingreso económico no es necesariamente una fuente de satisfacción en sí misma, el poder adquisitivo de las estudiantes para cubrir sus necesidades básicas podría ser un factor positivo de bienestar.
Asimismo, las adolescentes que reportaron una mala calidad de vida en amigos, vecindario y comunidad evidenciaron un nivel muy bajo de felicidad. Otros estudios confirman este hallazgo $(14,16,19)$. Una posible explicación es que en este grupo etario la presencia de los amigos en el barrio o comunidad se considera importante, debido a la necesidad que expresa el adolescente de pertenecer a un grupo. Sin embargo, experimentar hostilidad, envidia o rechazo por parte de los pares puede ocasionar un desajuste emocional en el adolescente $y$ por ende experiencias de infelicidad (20). 
Tabla 2. continuación

\begin{tabular}{|c|c|c|c|c|c|c|}
\hline \multicolumn{7}{|c|}{ Felicidad } \\
\hline & $\begin{array}{c}\text { Muy baja } \\
\text { felicidad } \\
\text { n }(\%)\end{array}$ & $\begin{array}{c}\text { Baja } \\
\text { felicidad } \\
\text { n }(\%)\end{array}$ & $\begin{array}{c}\text { Felicidad } \\
\text { media } \\
\text { n }(\%)\end{array}$ & $\begin{array}{c}\text { Alta } \\
\text { felicidad } \\
\text { n }(\%)\end{array}$ & $\begin{array}{c}\text { Muy alta } \\
\text { felicidad } \\
\text { n (\%) }\end{array}$ & $\mathrm{P}$ \\
\hline Educación y ocio & & & & & & 0.000 \\
\hline $\begin{array}{l}\text { Calidad de vida } \\
\text { óptima }\end{array}$ & $2(6.90)$ & $1(3.45)$ & $5(17.24)$ & $9(31.03)$ & $12(41.38)$ & \\
\hline $\begin{array}{l}\text { Tendencia a calidad } \\
\text { de vida buena }\end{array}$ & $20(20.00)$ & $10(10.00)$ & $35(35.00)$ & $15(15.00)$ & $20(20.00)$ & \\
\hline $\begin{array}{l}\text { Tendencia a baja } \\
\text { calidad de vida }\end{array}$ & $28(40.58)$ & $7(10.14)$ & $21(30.43)$ & $9(13.04)$ & $4(5.80)$ & \\
\hline Mala calidad de vida & $26(55.32)$ & $10(21.28)$ & $7(14.89)$ & $0(0.00)$ & $4(8.51)$ & \\
\hline Medios de comunicac & & & & & & 0.017 \\
\hline $\begin{array}{l}\text { Calidad de vida } \\
\text { óptima }\end{array}$ & $24(20.17)$ & $11(9.24)$ & $35(29.41)$ & $22(18.49)$ & $27(22.69)$ & \\
\hline $\begin{array}{l}\text { Tendencia a calidad } \\
\text { de vida buena }\end{array}$ & $25(37.31)$ & $8(11.94)$ & $21(31.34)$ & $8(11.94)$ & $5(7.46)$ & \\
\hline $\begin{array}{l}\text { Tendencia a baja } \\
\text { calidad de vida }\end{array}$ & $22(46.81)$ & $7(14.89)$ & $10(21.28)$ & $2(4.26)$ & $6(12.77)$ & \\
\hline Mala calidad de vida & $5(41.67)$ & $2(16.67)$ & $2(16.67)$ & $1(8.33)$ & $2(16.67)$ & \\
\hline Religión & & & & & & 0.001 \\
\hline $\begin{array}{l}\text { Calidad de vida } \\
\text { óptima }\end{array}$ & $5(13.16)$ & $2(5.26)$ & $13(34.21)$ & $8(21.05)$ & $10(26.32)$ & \\
\hline $\begin{array}{l}\text { Tendencia a calidad } \\
\text { de vida buena }\end{array}$ & $13(26.53)$ & $6(12.24)$ & $6(12.24)$ & $12(24.49)$ & $12(24.49)$ & \\
\hline $\begin{array}{l}\text { Tendencia a baja } \\
\text { calidad de vida }\end{array}$ & $31(32.63)$ & $10(10.53)$ & $30(31.58)$ & $11(11.58)$ & $13(13.68)$ & \\
\hline Mala calidad de vida & $27(42.86)$ & $10(15.87)$ & $19(30.16)$ & $2(3.17)$ & $5(7.94)$ & \\
\hline Salud & & & & & & 0.000 \\
\hline $\begin{array}{l}\text { Calidad de vida } \\
\text { óptima }\end{array}$ & $7(14.29)$ & $3(6.12)$ & $9(18.37)$ & $7(14.29)$ & 23(46.94) & \\
\hline $\begin{array}{l}\text { Tendencia a calidad } \\
\text { de vida buena }\end{array}$ & $21(24.42)$ & $6(6.98)$ & $26(30.23)$ & $20(23.26)$ & $13(15.12)$ & \\
\hline $\begin{array}{l}\text { Tendencia a baja } \\
\text { calidad de vida }\end{array}$ & $34(41.46)$ & $13(15.85)$ & $26(31.71)$ & $6(7.32)$ & $3(3.66)$ & \\
\hline Mala calidad de vida & $14(50.00)$ & $6(21.43)$ & $7(25.00)$ & $0(0.00)$ & $1(3.57)$ & \\
\hline
\end{tabular}

Tabla 3. Asociación entre felicidad y las variables sociodemográficas

\begin{tabular}{|c|c|c|c|c|c|c|}
\hline & $\begin{array}{c}\begin{array}{c}\text { Muy baja } \\
\text { felicidad }\end{array} \\
\text { n }(\%) \\
\end{array}$ & $\begin{array}{c}\text { Baja } \\
\text { felicidad } \\
\text { n }(\%)\end{array}$ & $\begin{array}{c}\text { Felicidad } \\
\text { media } \\
\text { n }(\%) \\
\end{array}$ & $\begin{array}{c}\text { Alta } \\
\text { felicidad } \\
\text { n (\%) }\end{array}$ & $\begin{array}{c}\text { Muy alta } \\
\text { felicidad } \\
\text { n }(\%)\end{array}$ & $\mathbf{P}$ \\
\hline $\begin{array}{l}\text { Con quienes } \\
\text { vive }\end{array}$ & & & & & & 0.017 \\
\hline Solo padre & $2(40.00)$ & $1(20.00)$ & $0(0.00)$ & $0(0.00)$ & $2(40.00)$ & \\
\hline Solo madre & $18(26.47)$ & $11(16.18)$ & $25(36.76)$ & $7(10.29)$ & $7(10.29)$ & \\
\hline Ambos padres & $23(27.71)$ & $8(9.64)$ & $19(22.89)$ & $20(24.10)$ & $13(15.66)$ & \\
\hline Solo familiares & $15(45.45)$ & $5(15.15)$ & $10(30.30)$ & $1(3.03)$ & $2(6.06)$ & \\
\hline $\begin{array}{l}\text { Padres y } \\
\text { familiares }\end{array}$ & $18(33.33)$ & $3(5.56)$ & $13(24.07)$ & $5(9.26)$ & $15(27.78)$ & \\
\hline Solo & $0(0.00)$ & $0(0.00)$ & $1(50.00)$ & $0(0.00)$ & $1(50.00)$ & \\
\hline
\end{tabular}

Además, las adolescentes que evidenciaron un nivel óptimo de vida familiar y familia extensa presentaron un nivel medio de felicidad. Otros estudios confirman este hallazgo $(14,16,21,22)$. Esto podría estar relacionado debido a que las adolescentes van aprendiendo que la felicidad se vive conforme se encuentran condiciones afectivas adecuadas, siendo la familia y la familia extensa un elemento básico de ello (22). Asimismo, para los adolescentes la familia es una red afectiva de 
sentimientos de cariño, de apoyo y de protección que le permite desarrollar estrategias de afrontamiento ante eventualidades externas negativas (22).

Las adolescentes que reportaron un nivel bueno de educación y ocio presentaron un nivel medio de felicidad. Otros estudios confirman este hallazgo $(16,23)$. Es decir, un ambiente adecuado en el colegio $y$ en el hogar que favorece la autorrealización propicia que el adolescente se encuentre consigo mismo, dedicándose a sus intereses, aficiones, a cumplir sus anhelos y proyectar su vocación (23). Por lo tanto, estas experiencias les producen felicidad y les permiten adquirir hábitos que posibiliten prácticas beneficiosas para sí mismos y la comunidad.

Las adolescentes que manifestaron un nivel óptimo en medios de comunicación evidenciaron un nivel medio de felicidad. De igual manera, otro estudio confirma este hallazgo (24). Es decir, los adolescentes que se muestran felices son aquellos que utilizan de forma adecuada los medios de comunicación para entretenerse. Una probable explicación es que los medios como el cine y la televisión son considerados agentes de socialización y son los adolescentes los consumidores más frecuentes, pues favorece en ellos el entretenimiento, la experiencia de nuevas sensaciones, la construcción de su identidad y además encuentran modelos de identificación a través de determinados valores e intereses (25).

Las adolescentes que reportaron un nivel bajo en religión presentaron un nivel medio de felicidad. De igual modo otros estudios coinciden con este hallazgo $(26,27)$. Una posible explicación es que los adolescentes, de acuerdo a su etapa de vida, priorizan otras actividades, adoptan un estilo de vida diferente y probablemente realicen pocas visitas a las iglesias independientemente de la afiliación religiosa. Por lo tanto, la baja religiosidad en ellos no es determinante para mantener sus niveles de felicidad (26).
Y en la última dimensión de la calidad de vida, las adolescentes que reportaron un nivel bajo en salud presentaron un nivel bajo de felicidad. Otros estudios confirman este hallazgo $(14,15,16,19)$. Una de las probables explicaciones, según el modelo biopsicosocial, es que la salud implica un equilibrio entre diversos factores como el biológico, psicológico, social y cultural, de manera que las adolescentes al no gozar de buena salud pueden desarrollar alguna enfermedad debido al desequilibrio en dichos factores, y por lo tanto, la felicidad se pudiera condicionar en función a su estado de salud (28).

Por otro lado, las estudiantes que viven solo con su progenitora presentaron niveles medios de felicidad. De igual manera, otros estudios confirman este hallazgo $(14,16,21,22)$. Una posible explicación es que el amor maternal representa probablemente la expresión afectiva de mayor calidez en comparación al padre y que existe reciprocidad de los hijos sobre todo hacia la madre (22). Asimismo, a pesar que en la etapa adolescente surgen inquietudes y nuevas relaciones sociales, la familia es el vínculo más importante para su desarrollo (29). Pues el saber que sus padres los aman, les aporta la seguridad de enfrentar las influencias negativas de su cultura y se sienten más optimistas (29).

Finalmente, es necesario mencionar las limitaciones del presente estudio, pues al ser de alcance correlacional no permitió establecer causalidad entre las variables. Además, el uso de instrumentos de auto-reporte en la recolección de la información podría haber presentado el sesgo de deseabilidad social en las adolescentes. Por otro lado, se resalta como fortaleza que el estudio sobre la felicidad y la calidad de vida en una población femenina es representativa de la situación sociocultural que se vive actualmente en las zonas centro y norte de Lima de las cuales provienen las estudiantes. 


\section{CONCLUSIONES}

Las adolescentes que manifestaron mala calidad de vida reportaron muy baja felicidad. Esto indica que, en determinados contextos, las adolescentes perciben que su deficiente calidad de vida interfiere con su estado de felicidad. Sin embargo, debido a la etapa en la que se encuentran de cambios biológicos, psicológicos y culturales, pueden variar notablemente su percepción. Puesto que, las adolescentes valoran la calidad de vida en función de sus experiencias en la familia, la salud, la vida social, los medios de comunicación y la religión que reflejan, en mayor o menor medida, su felicidad en dichos contextos.

Por consiguiente, de acuerdo a los resultados encontrados, se considera importante promover intervenciones dirigidas a potenciar sus recursos personales para favorecer el desarrollo óptimo de su calidad de vida. Asimismo, se debe considerar que las adolescentes se encuentran en la capacidad de valorar y analizar qué contextos son favorables o no para su desarrollo. Por lo cual, está en la iniciativa de los padres de familia y la comunidad educativa facilitar las condiciones para que ellas puedan expresarse y asumir su rol de participación en la mejora de su bienestar integral.

\section{REFERENCIAS BIBLIOGRÁFICAS}

1. Organización Mundial de la Salud. ur.nl Invertir en salud mental [Internet]. Ginebra, Suiza: OMS; 2004. 5. Helliwell J, Layard R, Sachs Disponible en: https://www.who.int/mental_healt h/advocacy/en/spanish_final.pdf

2. Domínguez B, Olvera Y. Estados 6. emocionales negativos dolor crónico y estrés. Revista ciencia [Internet]. 2006. 82(abril-junio): 66-75. Disponible en: http://www.revistacienciasunam.c om/es/53-revistas/revista-ciencias82/338-estados-emocionalesnegativos-dolor-cronico-yestres.html

3. Caycho Rodríguez, T. Alarcón, R. Psicología de la felicidad. 8. Grimaldo M. Calidad de vida en Introducción a la psicología positiva. Revista de Psicología [Internet]. 2010; 28(2):411-414. Disponible en: https://www.redalyc.org/pdf/3378/ 337829515008.pdf

4. Veenhoven. World Database of Happiness [Internet]. Países Bajos; 2019. Disponible en: http://worlddatabaseofhappiness.e
10. Calmeiro L, Camacho L, Camacho I, De Matos G. Life Satisfaction in Adolescents: The Role of Individual and Social Health Assets. The Sapanish journal of psychology. 2018; 21 . 2019.

Yamamoto. Felicidad a la Peruana [Internet]. Lima: Perú: Publimetro; $2013 . \quad$ Disponible en: https://publimetro.pe/actualida d/felicidad-peruana-11442-noticia/

7. Inga J, Vara A. Factores asociados a la satisfacción de vida de adultos mayores de 60 años en Lima-Perú. Universitas Psychologica. 2006; 5 (3):475-485. estudiantes de secundaria de la ciudad de Lima. Avances en psicología. 2012; 20(1):89-101. 14. Nava J. y Ureña J. Dominios y valoraciones sobre la felicidad en adolescentes de GuadalajaraMéxico: Revista Latinoamericana de Ciencias Sociales, Niñez y Juventud [Internet]. 2017; 15(1):443-454. Disponible en: http://www.scielo.org.co/pdf/rlcs/v 15n1/v15n1a29.pdf 
15. Vinaccia S, Quiceno J, Lozano F, 20. Silva A. Ser adolescente hoy. Romero S. Calidad de vida relacionada con la salud, percepción de enfermedad, felicidad y emociones negativas en pacientes con diagnóstico de artritis reumatoide. Acta Colombiana de Psicología. 2017; 20(1):49-59.

16. Oyanedel J, Inzunza J, Mella C. Bienestar subjetivo y calidad de vida en la infancia en Chile. Revista Latinoamericana de Ciencias Sociales, Niñez y Juventud [Internet]. 2015; 13(1):313-327. Disponible en: https://dialnet.unirioja.es/servlet/ar ticulo? codigo $=4999906$

17. Quiceno J, Vinaccia S. Calidad de vida en adolescentes: Análisis desde las fortalezas personales y las emociones negativas. Terapia psicológica. 2014; 32(3):185-200.

18. Gerónimo O, Valentín A, 23. López F, Sarrate M, Lebrero P. El Arancibia C, Campero S. Economía de la Felicidad: evidencia empírica para Latinoamérica. Revista Perspectivas. 2014; (34):159-180.

19. Higuita L, Cardona A. Concepto de calidad de vida en la adolescencia: una revisión crítica de la literatura. Revista CES Psicología [Internet]. 2015; 8(1):155-168. Disponible en: http://revistas.ces.edu.co/index.ph p/psicologia/article/view/3031/231 2

Fermentum. Revista Venezolana de Sociología y Antropología [Internet]. 2008; 18(52):312-332. Disponible http://www.redalyc.org/pdf/705/70 517520005.pdf

21. Méndez E. Relaciones intrafamiliares y la felicidad en los adolescentes del Colegio Unión de Naña 2018 [Tesis de Licenciatura]. Lima: Universidad Peruana Unión; 2019. Disponible en:

https://repositorio.upeu.edu.pe/bits tream/handle/UPEU/1799/Esther_ Tesis_Licenciatura_2019.pdf?sequ ence $=1 \&$ isAllowed $=\mathrm{y}$

22. Alarcón R. Funcionamiento familiar y sus relaciones con la felicidad. Revista Peruana de Psicología y Trabajo Social. 2017; 3(1):61-74.

ocio de los jóvenes en situación de vulnerabilidad. Análisis discursivo. Revista Española de Pedagogía [Internet]. 2016; 2(63):127-145. Disponible en: https://revistadepedagogia.org/wpcontent/uploads/2016/02/el-ociode-los-jovenes-1.pdf

24. Medrano C, Cortés A, Palacios S. Los valores personales y los 29. Chapman G. Cinco lenguajes del valores percibidos en la televisión: un estudio con adolescentes. Revista electrónica interuniversitaria de formación del profesorado [Internet]. 2009;
12(4):55-66. Disponible en: https://www.redalyc.org/pdf/2170/ 217014941005.pdf

25. Arnett J. Adolescent storm and stress, reconsidered. American Psychologist [Internet]. 1999; 54(5):317-326. Disponible en: http://jeffreyarnett.com/Arnett_19 99_AmPsy.pdf

26. Gómez M, Cogollo Z. Asociación entre religiosidad y estilo de vida en adolescentes. Revista de la Facultad de Medicina [Internet]. 2015; 63(2):193-198. Disponible en:

https://revistas.unal.edu.co/index.p $\mathrm{hp} / \mathrm{revfacmed} /$ article/view/49289

7. Tacilla S, Robles M. Felicidad y espiritualidad en miembros de una iglesia cristiana de Lima Este, 2015 [Tesis Licenciatura]. Lima: Universidad Peruana Unión; 2015. Disponible:

https://repositorio.upeu.edu.pe/han dle/UPEU/221

28. Oblitas L. Psicología de la salud: Una ciencia del bienestar y la felicidad. Avances en psicología [Internet]. 2008; 16(1):9-38. Disponible en: http://www.unife.edu.pe/pub/revps icologia/psicologiasalud.pdf amor, Los-Jóvenes: El secreto para amar a los jóvenes con eficacia. Ed. Unilit; 2017. 